\title{
Pelvic mass mimicking advanced tubo-ovarian malignancy with hepatic metastasis
}

A 49-year-old woman presented with mild pain in her lower abdomen and changes in bowel habits for 2 months. She also experienced $20 \mathrm{~kg}$ of weight loss over 7 months. She previously had an intrauterine device (IUD) for 5 years, removed 2 years prior to presentation. Vital signs were stable, and no fever was noted. Physical examination revealed a palpable pelvic mass without rebound pain. Laboratory examinations showed leukocytosis $\left(12,000 / \mathrm{mm}^{3}\right)$, anaemia (haemoglobin, $5.1 \mathrm{~g} / \mathrm{dL})$, elevated cancer antigen $(\mathrm{CA})-125(50.6 \mathrm{U} / \mathrm{mL})$, negative hepatitis B and hepatitis C serology, normal alpha-fetoprotein, and negative carcinoembryonic antigen. Sonography revealed 2 heterogeneous hypoechoic mass lesions in segment 8 of the liver measuring up to $7 \times 4 \mathrm{~cm}$. Computed tomography (CT) (Fig. 1) and magnetic resonance imaging (MRI) (Fig 2) were performed for further analysis.

What is your diagnosis?
A. Liver metastases from an adnexal malignancy
B. Lymphoma
C. Primary hepatic tumour
D. Actinomycosis
E. Tuberculosis

Findings and diagnosis. CT imaging revealed 4 distinct mixed solid and cystic hepatic masses, with the largest measuring $7.6 \times 5.9 \mathrm{~cm}$ in segment 8 of the liver. A solid and cystic mass with internal gas was identified in the right pelvis. The right ovary and sigmoid colon were difficult to distinguish separately from the mass. Diffuse peritoneal nodules, infiltration of the mesenteric fat, and bilateral hydronephrosis were also noted. Abdominal MRI without contrast, including T1- and T2-weighted images, showed a mixed cystic and solid mass in the liver. Central cystic component is of low T1-weighted and high T2-weighted signal intensity. Peripheral low signal intensity represents fibrosis, which is of low signal intensity on both $\mathrm{T} 1$ and T2-weighted images. Surrounding high signal on the T2 images was felt to be most consistent with oedema.

The patient underwent a complicated abdominal hysterectomy, bilateral salpingo-oophorectomy, adhesiolysis/enterolysis, liver biopsy, and bilateral Double-J stent placement for the hydronephrosis. The necrotic mass in the right adnexa measuring $6.5 \times 5 \times 4.5 \mathrm{~cm}$ was adherent to the surrounding pelvic structures, bowel loops, omentum and urinary bladder. The uterine corpus measured $12.5 \times 9.5 \times 5.0 \mathrm{~cm}$. The surface of the liver was adherent to the diaphragm, and a lesion measuring approximately $6 \times 7 \mathrm{~cm}$ in segment 8 of the liver was most consistent with abscess within the granulation tissue.

Histologic examination of the right ovary and fallopian tube yielded a diagnosis of tubo-ovarian abscess. Several sulfur granule-like particles were identified, confirming the diagnosis of actinomycosis infection.

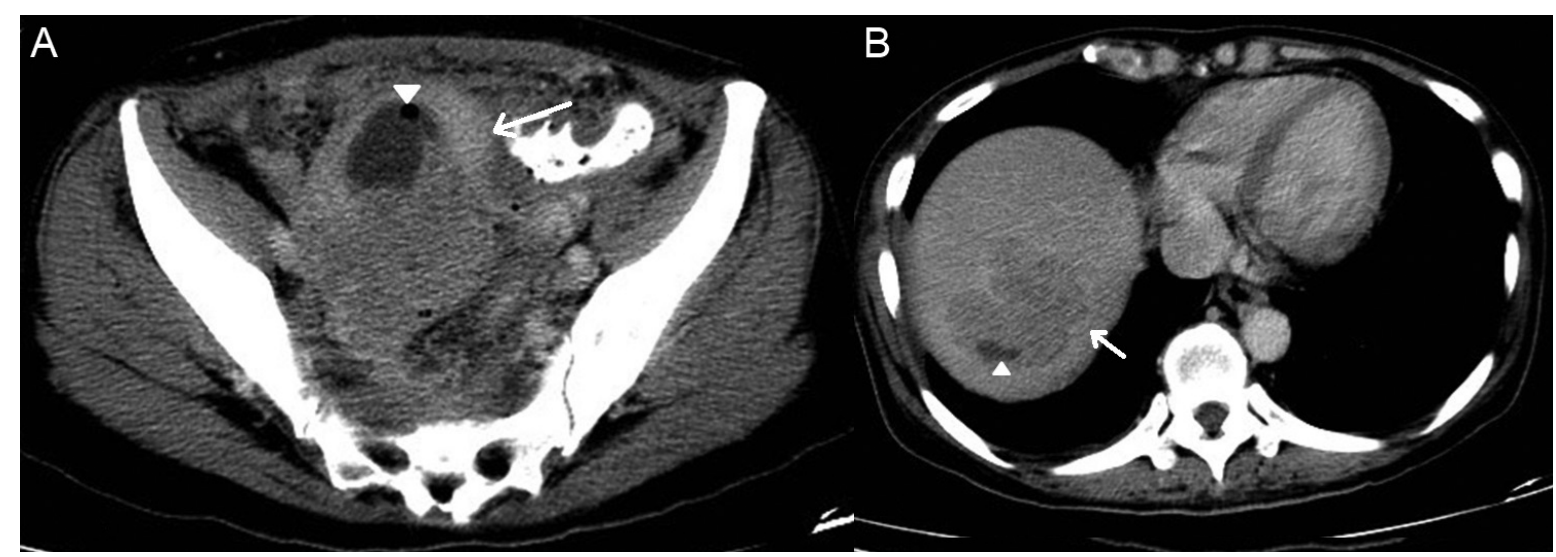

Fig. 1. Contrast-enhanced computed tomography scan obtained after intravenous administration of $100 \mathrm{~mL}$ of iomeprol (iohexol, $300 \mathrm{mg}$ of iodine $/ \mathrm{mL}$ ) showed (A) a heterogeneously enhancing soft tissue lesion (arrow) with a non-enhancing internal fluid collection and internal gas (arrowhead) in the right adnexa. (B) A mixed solid and cystic mass lesion (arrowhead) with peripheral faint contrast enhancement (arrow) in the liver dome was also appreciated. 

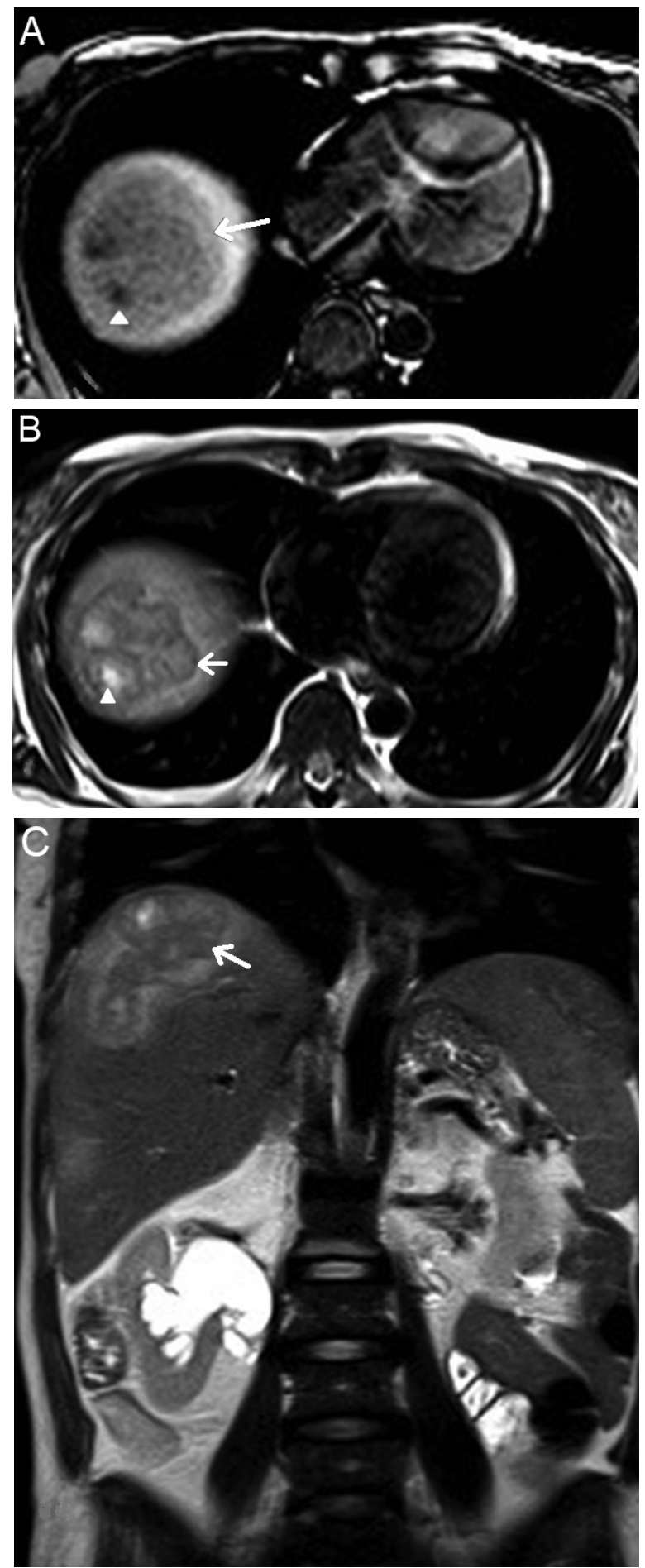

Fig. 2. (A) T1WI and (B) T2WI magnetic resonance imaging utilising a $1.5 \mathrm{~T}$ scanner without gadolinium administration; axial T1WI and coronal T2WI showed fibrosis rim of hepatic lesion (arrow) with low intensity, and an internal cystic component (arrowhead) with low intensity on T1WI and high intensity on T2WI. A peripheral rim of fibrosis was hypointense on both T1WI and T2WI (arrow). Surrounding T2 hyperintense oedema was noted. (C) Coronal T2WI showed a heterogeneously ill-defined high-signal-intensity soft tissue lesion in the right lobe of the liver (arrow) and right hydronephrosis.

T1WI: T1-weighted image; T2WI: T2-weighted image
The postoperative course was uncomplicated. A combination of metronidazole with ampicillin for management of intra-abdominal infected was given. The patient remained afebrile, and her pain resolved.

Discussion. Pelvic actinomycosis accounts for approximately $3-5 \%$ of actinomycosis cases, ${ }^{1}$ and it can be found in $1.65-11.60 \%$ of IUD users, even after IUD removal. ${ }^{2}$ The ovary and fallopian tube are most commonly affected. Haematogenous spread to the liver through the portal vein is uncommon, with approximately 4 cases having been reported since the case was reported in $2007 .^{3}$

Clinical signs and symptoms are nonspecific and can include weight loss, abdominal pain and general malaise. Laboratory data often reveal low-grade inflammation with leukocytosis and a positive C-reactive protein. Tumour marker values are normal to slightly elevated, as in malignancy. CT imaging findings include a pelvic mass containing a thick-walled cystic component and notably, internal gas, indicating infection with a gas-forming organism.

Hepatic actinomycosis manifested as an inflammatory pseudotumour in our patient. Additionally, perilesional dense fibrosis tends to be isointense to hypointense on T2-weighted MRI images, ${ }^{4}$ unlike metastatic tumour, which tends to show high signal on T2-weighted images. High signal intensity on diffusion-weighted images and hypointensity on apparent diffusion coefficient (ADC) maps are seen with both metastasis and abscess formation. However, mean ADC values for abscesses are significantly higher than for malignancies.

Primary or secondary hepatic lymphoma tends to be homogeneous and hypodense on CT, hyperintense on T2-weighted images and hypointense on T1-weighted images. Lymphoma also tends not to have a cystic component or a fibrotic rim. Hepatic calcification may indicate a tuberculosis granuloma. A multiloculated tubercular abscess with mild peripheral enhancement may mimic hepatic metastasis or other infection.

Histologic identification of gram-positive filamentous organisms with sulfur granules offers definitive diagnosis. The treatment of choice is beta-lactam antibiotics combined with a beta-lactamase inhibitor. ${ }^{5}$ Furthermore, surgical resection of the infected tissue may be necessary in complicated cases, especially in patients with a sinus tract, fistulae formation or necrotic tissue.

Conclusion. A history of IUD placement in patients with nonspecific clinical symptoms and a pelvic or abdominal mass should raise suspicion for actinomycosis in addition to malignancy. Delayed diagnosis may lead to death or require radical surgery. 


\section{REFERENCES}

1. Boyanova L, Kolarov R, Mateva L, et al. Actinomycosis: a frequently forgotten disease. Future Microbiol 2015;10:613-28.

2. McCormick JF, Scorgie RD. Unilateral tubo-ovarian actinomycosis in the presence of an intrauterine device. Am J Clin Pathol 1977; 68:622-6.

3. Morland D, Hassler S. Case 219: Pelvic Actinomycosis Mimicking Malignant Tumor. Radiology 2015;276:304-8.

4. Cheng YF, Hung CF, Liu YH, et al. Hepatic actinomycosis with portal vein occlusion. Gastrointest Radiol 1989;14:268-70.

5. Tan M, Lee JS, Pan JY. A Case of Recalcitrant Actinomycosis Unresponsive to Antibiotic Therapy. Ann Acad Med Singap 2016;45:475-6.
Yi-Chien Hsieh, ${ }^{1,2} M D$, Jerry Chin-Wei Chien, ${ }^{1,2} M D$, Tzu-I $\underline{\mathrm{Wu}},{ }^{3,4}{ }_{M D}$, Wing $\mathrm{P} \underline{\text { Chan }},{ }^{1,2} M D$

${ }^{1}$ Department of Radiology, Wan Fang Hospital, Taipei Medical University, Taipei, Taiwan

${ }^{2}$ Department of Radiology, School of Medicine, College of Medicine, Taipei Medical University, Taipei, Taiwan

${ }^{3}$ Department of Obstetrics and Gynecology, Wan Fang Hospital, Taipei Medical University, Taipei, Taiwan

${ }^{4}$ Department of Obstetrics and Gynecology, School of Medicine, College of Medicine, Taipei Medical University, Taipei, Taiwan

Correspondence: Dr Wing P Chan, Department of Radiology, Wan Fang Hospital, Taipei Medical University, 111 Hsing Long Road, Sec 3, Taipei 116, Taiwan.

Email: wingchan@tmu.edu.tw 